\title{
A new method for finding and characterizing galaxy groups via low-frequency radio surveys
}

\author{
J. H. Croston ${ }^{1 \star}$, J. Ineson ${ }^{2}$, M. J. Hardcastle ${ }^{3}$ and B. Mingo ${ }^{1}$ \\ ${ }^{1}$ School of Physical Sciences, The Open University, Walton Hall, Milton Keynes, MK6 7AA, UK \\ ${ }^{2}$ School of Physics and Astronomy, University of Southampton, Highfield, Southampton SO17 1BJ, UK \\ ${ }^{3}$ Centre for Astrophysics Research, School of Physics, Astronomy and Mathematics, University of Hertfordshire, Hatfield, Herts, AL10 9AB, UK.
}

Accepted XXX. Received YYY; in original form ZZZ

\begin{abstract}
We describe a new method for identifying and characterizing the thermodynamic state of large samples of evolved galaxy groups at high redshifts using high-resolution, low-frequency radio surveys, such as those that will be carried out with LOFAR and the Square Kilometre Array (SKA). We identify a sub-population of morphologically regular powerful (FRII) radio galaxies and demonstrate that, for this sub-population, the internal pressure of the radio lobes is a reliable tracer of the external intragroup/intracluster medium (ICM) pressure, and that the assumption of a universal pressure profile for relaxed groups enables the total mass and X-ray luminosity to be estimated. Using a sample of well-studied FRII radio galaxies, we demonstrate that our method enables the estimation of group/cluster X-ray luminosities over three orders of magnitude in luminosity to within a factor of $\sim 2$ from low-frequency radio properties alone. Our method could provide a powerful new tool for building samples of thousands of evolved galaxy groups at $z>1$ and characterizing their ICM.
\end{abstract}

Key words: galaxies: active $-\mathrm{X}$-rays: galaxies: clusters

\section{INTRODUCTION}

The thermodynamic evolution of baryons in forming and evolving galaxy groups and clusters at high redshift is not currently well constrained, but is an essential ingredient in cosmological studies of large-scale structure evolution (e.g. cluster counts, SunyaevZeldovich (SZ) studies, cosmic shear). With current X-ray telescopes it is just possible, with enormous expenditure of observing time, to identify a small number of evolved groups at $z>1$ (e.g. Tanaka et al. 2013; Finoguenov et al. 2015). SZ surveys are not currently sensitive to masses below $\sim 10^{14} \mathrm{M}_{\odot}$. Systematic studies of the evolution of the ICM above $z=1$ must therefore await next-generation instruments. New, reliable and efficient methods for identifying and characterizing evolved galaxy groups at high redshift would therefore be extremely valuable.

One of the major gaps in our understanding of ICM evolution is the cumulative contribution of AGN feedback from the protogroup/cluster stage to the present. The lack of observational constraints either on ICM properties or AGN feedback behaviour at high redshift is a limitation for cosmological simulations (e.g. McCarthy et al. 2017; Haider et al. 2016), and the relative contributions of quasar winds and jets are not well constrained. At present jet population demographics are poorly known beyond $z \sim 1$, but this situation is rapidly changing with first results emerging from the LOFAR extragalactic surveys, revealing the popula-

^ Email: Judith.Croston@open.ac.uk tion of low/intermediate luminosity jets at high redshift (e.g. Hardcastle et al. 2016; Williams et al. 2016; Shimwell et al. 2017). These new radio surveys have enormous potential to improve our understanding of AGN feedback, but their interpretation requires (1) a firm understanding of the physics and energetics of the diversity of jet sub-populations, so that radio properties can be translated into energetic impact, and (2) the ability to map jet populations to the dark matter haloes in which they live.

We have recently completed a comprehensive survey of the environments of radio-loud AGN, across three orders of magnitude in radio luminosity and environmental richness (Ineson et al. 2013, 2015, 2017). A key outcome of this work is the demonstration that both morphology (FRI/II class) and accretion mode (low/high excitation) influence the locations and impact of jet feedback. We have found a relationship between radio luminosity and cluster richness for low-excitation radio galaxies (LERGs), which holds across four orders of magnitude, with evidence that morphology is a source of scatter in this relation. No similar relationship was found for high-excitation radio galaxies (HERGs), which are found almost exclusively to occupy galaxy groups (at least in the redshift range of $z<0.6$ considered in these studies). Our work suggests that radio galaxies will be excellent indicators of galaxy group (rather than rich cluster) environments at high redshift, but the LERG $L_{\mathrm{R}}-$ $L_{\mathrm{X}}$ relation we have found (Ineson et al. 2015) (where $L_{\mathrm{R}}$ is lowfrequency radio luminosity and $L_{\mathrm{X}}$ is bolometric X-ray luminosity) has large scatter. In addition, the radio-loud AGN population is likely to become increasingly dominated by high-excitation sources 
at high redshift (e.g. Best et al. 2014; Williams \& Röttgering 2015; Williams et al. 2016). Radio luminosity alone therefore does not provide a reliable indicator of a galaxy group environment or allow the prediction of halo mass or X-ray luminosity to within an order of magnitude.

The aim of this paper is to present a new method of identifying and characterizing evolved galaxy group environments, based on considering the AGN sub-population whose internal physical conditions are best understood. We have shown (Croston et al. 2005; Ineson et al. 2017) via X-ray inverse-Compton (IC) observations of radio lobes, that FRII radio galaxies have typical magnetic field strengths $(B)$ a factor of a few less than the equipartition value ( $B_{\text {eq }}$, e.g. Burbidge 1956) calculated with the assumption of no protons. The FRII radio galaxies in the representative sample of Ineson et al. (2015) have well-constrained internal pressures from $\mathrm{X}$-ray IC measurements and external pressures from the ICM Xray emission (Ineson et al. 2017, hereafter I17) allowing us to examine the source dynamics and establish that FRII lobes do not contain a significant proton population. This result is in contrast to the inferred internal conditions in low-power (FRI) radio galaxies, where it is well established that significant proton content and/or departure from equipartition is required for pressure balance (e.g. Croston et al. 2003, 2008; Bîrzan et al. 2008; Croston \& Hardcastle 2014). Our FRII study has led us to postulate that - for the FRII jet sub-population only - the radio-measured internal pressure should provide a reliable estimate of the external ICM pressure at the lobe midpoint, where we have found that the lobes are close to pressure balance with the environment (consistent with hydrodynamical simulations of FRII lobe evolution, e.g. Hardcastle \& Krause 2014).

Below we describe a method to exploit these results, and demonstrate that FRII radio galaxy samples from wide-area lowfrequency radio surveys (including both low- and high-excitation objects) can be used to find and characterize evolved galaxy groups, potentially enabling the compilation of large catalogues of groups at high redshift.

\section{A METHOD FOR PREDICTING GROUP-SCALE ENVIRONMENTS}

Our aim is to demonstrate that group/cluster X-ray luminosity can be predicted from radio properties alone. Specifically, for regular FRII radio galaxies the relatively narrow distribution of both $B_{\text {obs }} / B_{\text {eq }}$ and midpoint $P_{\text {ext }} / P_{\text {int }}$ (I17) leads us to expect that the radio-estimated internal pressure should be a reliable predictor of external ICM pressure, which can then be used to estimate the overall environmental richness. In order to have a robust method that can be applied to large-area surveys, we need to be able to use easily measured radio properties to identify the subpopulation of radio-loud AGN for which such predictions are likely to be reliable. Below we discuss how to select such a sample, before developing and testing a radio-based method for predicting ICM luminosity using the sample of I17.

\subsection{Morphological selection}

The sub-sample of radio galaxies whose large-scale environments should be predictable from radio-lobe pressure estimates must meet the following requirements:

- FRII (edge-brightened) morphology, so that proton content is expected to be minimal (Croston et al. 2005; Ineson et al. 2017)
- symmetric "classical double" structure, which we postulate should be indicative of a relaxed environment with the radio galaxy located at the centre of the dark matter halo (while disturbed or asymmetric lobe morphologies indicate more dynamic/irregular gas distributions)

- low-frequency lobe emission that extends a substantial distance from the hotspots back towards the nucleus, so that the internal pressure can be estimated reliably without hotspot contamination.

By applying these criteria, we expect to select radio galaxies for which the magnetic field strength relative to equipartition and lobe overpressuring at the midpoint are similar.

The FRII sample of I17 meets the first criterion listed above. We applied the second and third criteria to this sample, initially using our high-resolution radio maps described in Ineson et al. (2013) and Ineson et al. (2015). The parent sample of I17 includes 33 radio galaxies with measured external pressure distributions, in two redshift ranges, $z<0.2$ and $0.4<z<0.6$. Applying the selection criteria above led to the exclusion of 6 objects: 3C 303, 3C 321 $3 \mathrm{C} 433,4 \mathrm{C} 73.08$, 6C 0850+3747, and 7C 0219+3423. The remaining sources all have regular, symmetric "classical double" morphology and extended lobes, without significant signs of disturbance. Therefore, in this redshift range at least $\sim 80$ per cent of FRIIs are good candidates for our method. Fig. 1 shows examples of sources excluded due to being dominated by hotspot emission or having an irregular morphology. In the following sections we make use of our morphologically regular subsample of 27 objects, which spans three orders of magnitude in radio luminosity from $10^{25} \mathrm{~W} \mathrm{~Hz}^{-1}$ $\mathrm{sr}^{-1}<L_{151 \mathrm{MHz}}<10^{28} \mathrm{~W} \mathrm{~Hz}^{-1} \mathrm{sr}^{-1}$ and contains objects with linear sizes between $50-1000 \mathrm{kpc}$. While the ratio of regular to asymmetric/disturbed FRIIs may evolve with redshift, we nevertheless expect to be able to construct very large samples of objects meeting our criteria from radio surveys. In Section 3 we comment on how well our morphological selection criteria can be applied to low-resolution radio surveys. We note that we make no selection based on accretion mode (low- or high-excitation class), as I17 have shown that there is no difference in the lobe composition or dynamics of FRII LERGs and HERGs.

\subsection{Predicting the external pressures around FRII radio lobes}

We used the sample of 27 FRII radio galaxies obtained from the selection of Section 2.1 to investigate how well the external ICM pressure could be predicted from the radio measurements alone. Our assumption is that because the distribution of magnetic field strengths for the FRII population is narrow, and the contribution of protons is minimal (I17, particularly figures 1 and 3 of that work), we can obtain a reasonable estimate of the internal pressure by assuming a magnetic field strength of $B=0.4 B_{\text {eq }}$, which is the median value found in the IC analysis of I17.

For each radio galaxy we used the measured source geometry and radio flux measurements from I17 to model the internal pressure on the assumption of $B=0.4 B_{\text {eq }}$ and no protons. We used the SYNCH code of Hardcastle et al. (1998) to determine the lobe internal pressures, assuming a power law in electron energy between $\gamma_{\min }=10$ and $\gamma_{\max }=10^{5}$, with an energy index of $\delta=2.4$ (as discussed by I17). This method can be applied to large samples obtained from low-frequency surveys, with no need for additional information from other wavelengths other than the source redshift, 

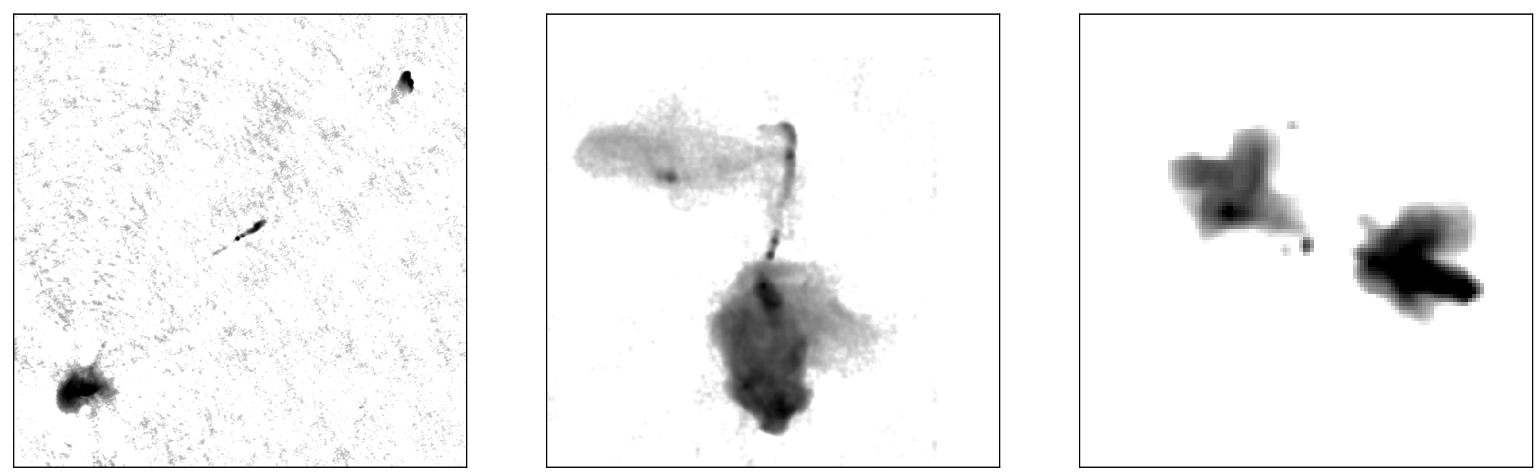

Figure 1. Examples of sources excluded from the sample due to irregular morphology or lack of extended lobe emission at low frequencies, from left: $3 \mathrm{C} 321$, 3C 433 and 4C 73.08. Images were obtained from the Atlas of DRAGNs (Leahy, Bridle \& Ström: http://www. jb.man.ac.uk/atlas/).
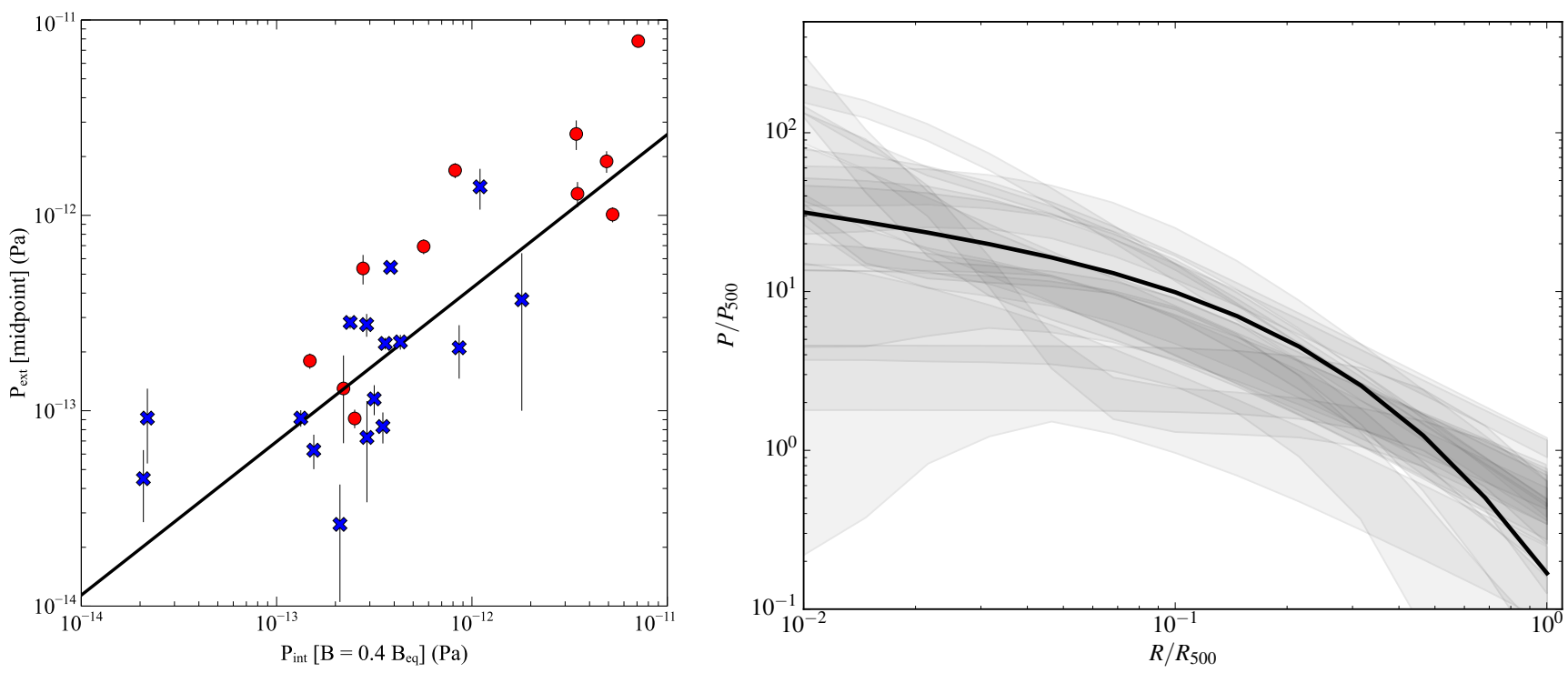

Figure 2. Left: The internal lobe pressures calculated assuming $B=0.4 B_{\mathrm{eq}}$ vs. the measured external ICM pressure at the mid-lobe distance, for the morphologically selected subsample of I17, demonstrating that this radio-only pressure estimate can reliably predict the external mid-lobe pressure (solid line is the best-fitting relation as given by Eq. 1$)$. Blue crosses indicate the low- $z$ sample $(z<0.2)$ and red plus symbols the high-z sample $(0.4<z<0.6)$, showing that the same relation holds for both subsamples, which implies that the relation is not driven by a distance dependence. Right: External pressure profiles for the subsample of I17 environments with well-constrained temperatures indicated by grey shaded areas showing $1 \sigma$ credible intervals. The universal profile of Arnaud et al. (2010) is plotted as a solid black line.

provided that the source geometry and lobe radio flux can be measured reliably.

We compared our radio-estimated internal pressures $\left(P_{\text {int }}\right)$ with the measured external (ICM) pressure for each source at the midpoint of the modelled radio lobe $\left(P_{\text {ext }}\right)$, as shown in Fig. 2 . We find that our radio-estimated internal lobe pressures are well correlated with the external mid-lobe pressures, consistent with the finding of I17 that the lobes are typically close to pressure balance at their midpoints. We used an MCMC least squares fitting code written using the EMCEE package (Foreman-Mackey et al. 2013) to determine the best-fitting relation between our estimated values of $P_{\text {int }}$ and measured $P_{\text {ext }}$. Our best-fitting relation, with 90 per cent confidence intervals, is:

$\log _{10} P_{\text {ext }}=0.79_{-0.03}^{+0.02} \log _{10} P_{\text {int }}-2.9 \pm 0.3$

We find a slope a little lower than unity, which is consistent with a pressure ratio that has some dependence on the internal pressure. This is physically plausible as sources at high pressure may be expected to be more overpressured even at the midpoint. We also carried out the same comparison using the external pressure further out at the lobe tip instead of the midpoint; however, we found that the internal pressure is a much weaker predictor of the lobe-tip pressure, which is as expected since I17 found a much wider range of external-to-internal pressure ratios at the lobe tip.

Fig. 2 shows that there is no strong evolution of the best-fitting pressure relation with redshift: a Mann-Whitney U test of the distributions about this relation does not rule out at 95 per cent confidence the null hypothesis that the two redshift subsamples have the same parent population. We do, however, acknowledge that weak evolution of the relation of Eq. 1 cannot be ruled out by our data, and this should be investigated with deep X-ray surveys data and/or further deep X-ray observations of carefully selected samples. 

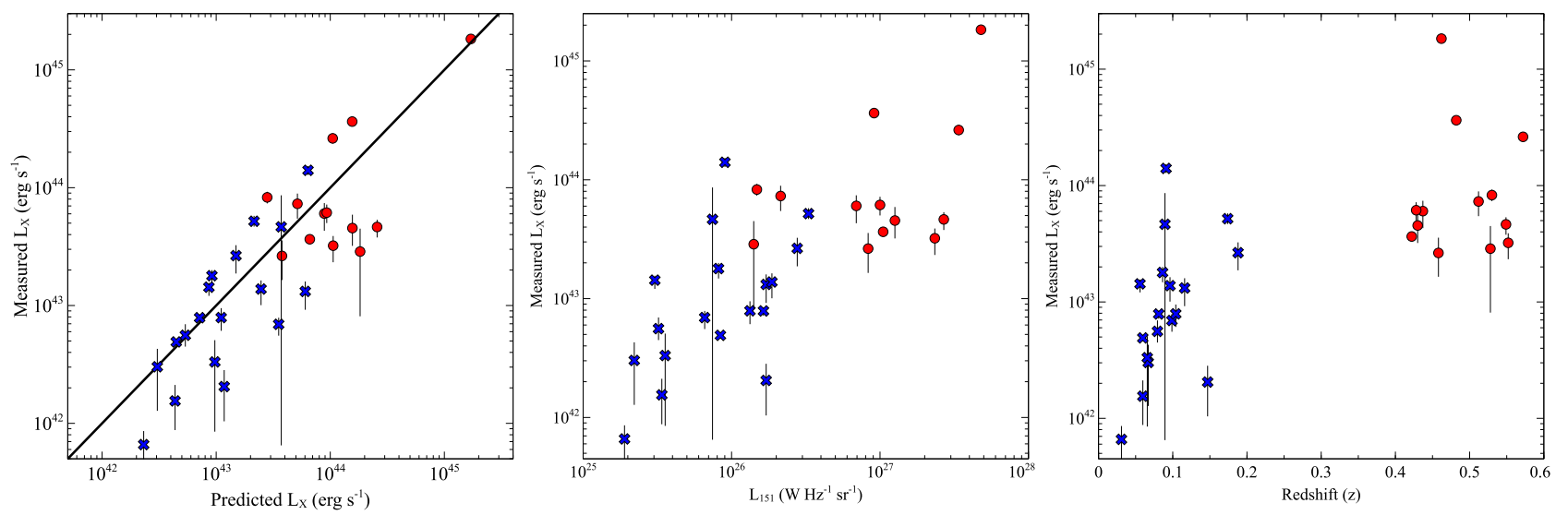

Figure 3. Left: The predicted group/cluster X-ray luminosities determined from the radio-measured pressures for the morphologically selected subsample of I17, obtained using the method described in Section 2.3, compared with their measured X-ray luminosities, demonstrating that for this sample the environments can reliably be predicted from the (low-frequency) radio-estimated lobe pressures. A line of unity (not best fit) is shown as a solid line. Colours and symbols are as for the previous figure, showing that the prediction method is successful for both redshift ranges. Middle: The relationship between measured X-ray luminosity and radio luminosity, and right: the relationship between measured X-ray luminosity and redshift.

\subsection{Using pressure to predict X-ray luminosity}

We have demonstrated that we can reliably predict the ICM pressure at a single distance within the group/cluster surrounding an FRII radio galaxy. To be able to predict the overall group/cluster richness, we need to be able to translate this pressure measurement into an estimate of cluster mass and/or X-ray luminosity. We therefore examined whether the universal cluster pressure profile of Arnaud et al. 2010 (hereafter A10) could be applied to FRII environments. We first examined the subsample of I17 for which reliable spectral temperature estimates were available. Our pressure profiles have large uncertainties, and were obtained from either beta or double-beta model fits to the surface-brightness profiles (see I17). The environments are also mainly galaxy groups $\left(L_{\mathrm{X}}<10^{44} \mathrm{erg}\right.$ $\mathrm{s}-1$ ), and so lower in mass than the sample of A10; however, Sun et al. (2011) have shown that the universal profile also applies on galaxy group scales. Fig. 2 (right) shows that our scaled pressure profiles are in broad agreement with the A10 profile shape and normalization.

We therefore tested whether the universal cluster pressure profile could be used to translate our single-radius pressure estimates, from our radio predictions, into cluster mass and X-ray luminosity. We used the profile of A10 (Equations 11, 12 and 13 of that work) and our input values of distance and $P_{\text {ext }}$ to estimate $M_{500}$ for each radio-galaxy environment. We then applied the $L_{\mathrm{X}}-M_{500}$ relation of Pratt et al. (2009) (using the relation for $L_{\mathrm{X}}<R_{500}$ ) to obtain a predicted $L_{\mathrm{X}}$ for each radio-galaxy environment. Finally we compared our radio-predicted $X$-ray luminosities with the measured $X$ ray luminosities for our sample from Ineson et al. (2015). Fig. 3 (left) shows the remarkable agreement between our radio-predicted $\mathrm{X}$-ray luminosities and the measured values across more than three orders of magnitude in X-ray luminosity (from poor groups to moderate clusters) - our method appears to predict ICM X-ray luminosity to within roughly a factor of two based only on the measured properties of the embedded radio galaxy. We have included four additional FRII sources from the sample of I17 for which external pressure distributions could not be measured, but which meet the morphological selection criteria, leading to a sample of 31 objects, as listed in Table 1.

As shown in the middle panel of Fig. 3, there is a correlation in our sample between the much simpler quantity of radio luminosity and X-ray luminosity, which could also be used to predict FRII galaxy group environments; however, this has much greater scatter (more than an order of magnitude), and thus weaker predictive power. Our sample does have a relationship between distance and measured X-ray luminosity. We therefore show the low- $z(z<0.2)$ and high- $z(0.4<z<0.6)$ subsamples of I17 in different colours in Figs. 2 and 3 to demonstrate that the midpoint pressure relation and the predictive method both apply separately to the two subsamples. The right-hand panel of Fig. 3 also demonstrates clearly that there is no significant distance dependence of X-ray luminosity within each subsample, and hence the strong predictive relation of the left-hand panel cannot be driven by distance.

\section{DISCUSSION}

Our method of predicting environmental X-ray luminosity based on radio properties alone, presented in the previous section, appears extremely successful; however, there are a number of questions that must be addressed in order to be confident that the method will be useful for large samples at higher redshift.

We must first consider how well our radio selection procedure can be applied to data from wide-field radio surveys. In the shortterm, we hope to apply our method to the LOFAR Tier-1 surveys data (Shimwell et al. 2017), which has a spatial resolution of $\sim 6$ arcsec, corresponding to a physical resolution of $\sim 50 \mathrm{kpc}$ at $z=1$. We re-applied our morphological selection criteria using the TGSS Alternative Data Release (ADR) (Intema et al. 2017) 150-MHz images for the 117 parent sample, which have a typical resolution of $\sim 25$ arcsec, so that the physical resolution at $z \sim 0.1$ is comparable to LOFAR Tier- 1 resolution at $z \sim 1$. We found that $28 / 33$ sources were sufficiently resolved to apply the criteria of $\S 2.1$, with all of the sources we originally excluded using the high-resolution data also being excluded using TGSS. A further five sources were excluded due to inadequate resolution, so that our method could have been applied to a sample of 23 objects had we only had data of TGSS resolution available. We therefore conclude that our morphological selection method can be applied accurately to LOFAR surveys out to $z>1$. 
We used the LOFAR Boötes Tier-1 survey data presented by Williams et al. (2016) and Williams et al. (2017) to estimate the sky density of FRII radio galaxies at high redshifts. We take the assumption that all AGN above $L_{150 \mathrm{MHz}}=3 \times 10^{26} \mathrm{~W} \mathrm{~Hz}^{-1}$ are FRIIs, leading to estimated sky densities of $\sim 1.5$ FRIIs deg ${ }^{2}$ between $0.5<z<1, \sim 2$ FRIIs $\operatorname{deg}^{2}$ between $1<z<1.5$ and $\sim 1$ FRIIs $\mathrm{deg}^{2}$ between $1.5<z<2$. The resolved fractions are 100,90 and 82 per cent, respectively, for the three redshift bins (Williams, private communication). The adopted luminosity cut is conservative, as it is now known that sources of FRII morphology can be found at luminosities well below the traditional Fanaroff \& Riley (1974) divide (e.g. Miraghaei \& Best 2017), hence the true numbers could be higher. However, it is also known that source sizes in flux-limited samples evolve with redshift (e.g. Blundell et al. 1999) which could reduce the sample sizes that are classifiable with LOFAR data. Such size evolution is seen in a subset of the LOFAR Boötes sample examined by Morabito et al. (2017), but their sample excluded low-excitation radio galaxies, and included only objects with firm spectroscopic redshifts, which may introduce host galaxy/environmental biases: the size evolution to $z=2$ of the full LOFAR 150-MHz selected population is not yet well determined. If we assume, based on our TGSS comparison and consistent with the LOFAR-Boötes resolved fraction, that $\sim 2 / 3$ of the high-redshift sources will meet our morphological classification criteria, we would expect to be able to compile samples of $>5,000$ candidate galaxy groups at $z>1$, and $>2,000$ at $z>1.5$ with LOFAR Tier-1 surveys over the next few years, as the observed sky area accumulates ${ }^{1}$. Size evolution may reduce these sample sizes by a factor of a few, but large numbers of $>200-\mathrm{kpc}$ sized objects should be detectable in this redshift range (e.g. $\sim 1 / 3$ of 3 CRR objects at $z>1$ have linear sizes greater than $200 \mathrm{kpc}$ ). With the substantially higher resolution and sensitivity expected to be available with the SKA, it should be possible to extend the method to the redshifts where the earliest galaxy groups with a hot ICM are forming.

Of course another important concern is that our method relies on the applicability of the universal pressure profile of A10 to FRII radio-galaxy environments over a large redshift range. We have demonstrated in Section 2.2 that our sample of radio-galaxy environments at $z<0.6$ have pressure profiles broadly consistent with A10; however, our method will be most valuable if it can be applied to environments at $z>1$ where methods to identify and characterize group-scale environments in particular are very limited. A crucial question is therefore whether we would expect our method to apply at all redshifts. Since the radio lobes themselves are sensitive to changes in the typical pressure distribution, if the typical ICM environment for FRII radio galaxies evolves with redshift our method should nevertheless accurately predict the external pressure at the lobe midpoint. If the gas distributions of FRII environments evolve to become typically more dynamic/irregular at $z>1$ then we would expect that a larger fraction of the FRII population will fail to meet our morphological selection criteria; however, those that do would be expected to occupy relaxed gas distributions. We must then ask whether the universal pressure profile of A10 should continue to predict $M_{500}$ correctly at $z>1$. As the formulae we use account for standard evolution (e.g. of $\rho_{\text {crit }}$ ) with redshift we would expect the prediction of $M_{500}$ to be reliable. A more serious concern is whether we would expect to see either a bias or increased scatter in the relationship between $M_{500}$ and

1 Around 2,500 $\mathrm{deg}^{2}$ of mainly contiguous sky area has been observed to Tier-1 depth as of May 2017
$L_{\mathrm{X}}$. The relationship of Pratt et al. (2009) is for clusters at $z<0.2$; our method includes standard self-similar evolution, but the evolution of this relation at $z>1$ is not observationally tested. Any systematic departure from this form of evolution, and particularly any increased scatter in the relation between $L_{\mathrm{X}}$ and $M_{500}$ in forming proto-groups and clusters will affect our method.

While the caveats above show the need for some caution in applying our method to high redshifts, it is important to emphasize that the radio surveys that will provide the samples of high-redshift FRIIs for environmental characterization should offer strong clues about the effects of any environmental evolution: any systematic changes in the environments of the FRII radio galaxies will also be manifest as changes in the size, $L_{\mathrm{R}}$ and morphological distributions as a function of redshift. It will be necessary to disentangle such effects from changes caused by other effects, such as evolution in AGN duty cycles; however, comparisons with hydrodynamical simulations of FRII radio galaxies in a range of environments (e.g. Hardcastle \& Krause 2014; English et al. 2016) should enable careful diagnosis of the range of environments at $z>1$.

We therefore conclude that our method offers considerable potential for the compilation of large samples of galaxy groups at $z>1$ with LOFAR and out to $z>2$ with future surveys such as those planned with the SKA. It will be essential to calibrate our method rigorously at moderate redshifts with X-ray observations of new samples of radio galaxies with unknown large-scale environments. If this is successful then we will be able to compile large galaxy group catalogues via embedded FRII radio galaxies using LOFAR surveys data over the next few years.

\section{SUMMARY}

We have described a new method by which embedded FRII radio galaxies can be used to characterize group and cluster environments, which offers the potential to compile large catalogues of evolved galaxy groups at $z>1$. Our method exploits two important observational results applying to the sub-population of 'classical double' FRII radio galaxies alone:

- the conclusion from X-ray inverse-Compton studies that FRII radio galaxies have a narrow distribution in magnetic field strength, with a median value of $0.4 \times$ the equipartition field strength, and contain no energetically important protons.

- the conclusion from comparisons of external and internal pressures that FRII lobes are typically within a small factor of pressure balance at the lobe midpoint

These two results allow us to estimate reliably the internal radiolobe pressures of morphologically regular FRII radio galaxies and therefore to estimate the external ICM pressure at the lobe midpoint from low-frequency radio measurements alone. In particular, because our method is based on the lobe composition and dynamics, it applies to both low and high-excitation FRII radio galaxies.

We have tested our method by applying a simple morphological selection to the FRII sample of I17, demonstrating that the Xray luminosity of the host galaxy group or cluster can be predicted to within a factor of $\sim 2$. It will be necessary to test our method carefully using new samples of radio galaxies with unknown environments, calibrating any evolutionary effects not accounted for by the current analysis; however, radio-lobe morphology is a sensitive probe of environmental gas distributions, and hence applying careful morphological selection to high redshift samples is likely to prove an effective means of finding, and characterizing the ICM 
of, large samples of galaxy groups and low-mass clusters. If such samples can be compiled they will be both of general value in the study of large-scale structure evolution and specifically useful for studies of radio-loud AGN feedback at high redshift.

\section{ACKNOWLEDGMENTS}

JHC, BM and MJH acknowledge support from the Science and Technology Facilities Council (STFC) under grants ST/M001326/1 and ST/M001008/1. We would like to thank Wendy Williams for providing unpublished information about AGN resolved fractions for the LOFAR-Boötes catalogue.

\section{REFERENCES}

Arnaud M., Pratt G. W., Piffaretti R., Böhringer H., Croston J. H., Pointecouteau E., 2010, A\&A, 517, A92

Best P. N., Ker L. M., Simpson C., Rigby E. E., Sabater J., 2014, MNRAS, 445,955

Bîrzan L., McNamara B. R., Nulsen P. E. J., Carilli C. L., Wise M. W., 2008, ApJ, 686, 859

Blundell K. M., Rawlings S., Willott C. J., 1999, AJ, 117, 677

Burbidge G. R., 1956, ApJ, 124, 416

Croston J. H., Hardcastle M. J., 2014, MNRAS, 438, 3310

Croston J. H., Hardcastle M. J., Birkinshaw M., Worrall D. M., 2003, MNRAS, 346, 1041

Croston J. H., Hardcastle M. J., Harris D. E., Belsole E., Birkinshaw M., Worrall D. M., 2005, ApJ, 626, 733

Croston J. H., Hardcastle M. J., Birkinshaw M., Worrall D. M., Laing R. A., 2008, MNRAS, 386, 1709

English W., Hardcastle M. J., Krause M. G. H., 2016, MNRAS, 461, 2025

Fanaroff B. L., Riley J. M., 1974, MNRAS, 167, 31P

Finoguenov A., et al., 2015, A\&A, 576, A130

Foreman-Mackey D., Hogg D. W., Lang D., Goodman J., 2013, PASP, 125, 306

Haider M., Steinhauser D., Vogelsberger M., Genel S., Springel V., Torrey P., Hernquist L., 2016, MNRAS, 457, 3024

Hardcastle M. J., Krause M. G. H., 2014, MNRAS, 443, 1482

Hardcastle M. J., Birkinshaw M., Worrall D. M., 1998, MNRAS, 294, 615

Hardcastle M. J., et al., 2016, MNRAS, 462, 1910

Ineson J., Croston J. H., Hardcastle M. J., Kraft R. P., Evans D. A., Jarvis M., 2013, ApJ, 770, 136

Ineson J., Croston J. H., Hardcastle M. J., Kraft R. P., Evans D. A., Jarvis M., 2015, MNRAS, 453, 2682

Ineson J., Croston J. H., Hardcastle M. J., Mingo B., 2017, MNRAS, 467, 1586

Intema H. T., Jagannathan P., Mooley K. P., Frail D. A., 2017, A\&A, 598, A78

McCarthy I. G., Schaye J., Bird S., Le Brun A. M. C., 2017, MNRAS, 465, 2936

Miraghaei H., Best P. N., 2017, MNRAS, 466, 4346

Morabito L. K., et al., 2017, preprint, (arXiv: 1704.06267)

Pratt G. W., Croston J. H., Arnaud M., Böhringer H., 2009, A\&A, 498, 361

Shimwell T. W., et al., 2017, A\&A, 598, A104

Sun M., Sehgal N., Voit G. M., Donahue M., Jones C., Forman W., Vikhlinin A., Sarazin C., 2011, ApJ, 727, L49

Tanaka M., et al., 2013, PASJ, 65, 17

Williams W. L., Röttgering H. J. A., 2015, MNRAS, 450, 1538

Williams W. L., et al., 2016, MNRAS, 460, 2385

Williams W., et al., 2017, MNRAS, submitted.

This paper has been typeset from a $\mathrm{T}_{\mathrm{E}} \mathrm{X} / \mathrm{L}_{\mathrm{A}} \mathrm{X}$ file prepared by the author. 
Table 1. Table of source properties for the I17 morphologically regular subsample. Fields are: source name, redshift, 151-MHz radio luminosity, lobe spherical radius (the radius of a sphere with equivalent volume to one lobe - average for the two lobes), midpoint distance, estimated internal pressure (assuming $B=0.4 B_{\mathrm{eq}}$ ), measured external pressure at the midpoint, and measured bolometric X-ray luminosity for $R<R_{500}$. All $\mathrm{X}$-ray measurements and source geometries are taken from I17; redshifts and radio luminosities are as compiled from the literature by Ineson et al. (2015). Note that four sources, not included in Fig. 2, do not have external pressure measurements, as explained in the text.

\begin{tabular}{|c|c|c|c|c|c|c|c|}
\hline Source & $z$ & $\begin{array}{r}L_{151 M H z} \\
10^{25} \mathrm{~W} \mathrm{~Hz} \mathrm{sr}^{-1}\end{array}$ & $\begin{array}{r}\text { Lobe radius } \\
\mathrm{kpc}\end{array}$ & $\begin{array}{r}\text { Midpoint } \\
\mathrm{kpc}\end{array}$ & $\begin{array}{r}P_{\text {int }} \\
10^{-13} \mathrm{~Pa}\end{array}$ & $\begin{array}{r}P_{\text {ext,mid }} \\
10^{-13} \mathrm{~Pa}\end{array}$ & $\begin{array}{r}L_{X} \\
10^{43} \mathrm{erg} \mathrm{s}^{-1}\end{array}$ \\
\hline $3 C 19$ & 0.482 & 91.2 & 10.2 & 11.0 & 340 & - & $36 \pm 3$ \\
\hline $3 \mathrm{C} 33$ & 0.0596 & 8.4 & 59 & 86.4 & 2.1 & $0.26_{-0.23}^{+0.8}$ & $0.49_{-0.03}^{+0.02}$ \\
\hline $3 C 35$ & 0.067 & 2.2 & 185 & 239 & 2.1 & $0.45_{-0.21}^{+0.15}$ & $0.3_{-0.17}^{+0.13}$ \\
\hline $3 C 46$ & 0.437 & 69 & 167 & 293 & 2.8 & $5.4_{-0.7}^{+1.1}$ & $6.0_{-1.7}^{+1.4}$ \\
\hline $3 \mathrm{C} 98$ & 0.309 & 1.9 & 41 & 42.6 & 2.9 & $0.73_{-0.5}^{+0.4}$ & $0.07 \pm 0.02$ \\
\hline $3 \mathrm{C} 192$ & 0.0597 & 3.4 & 64 & 63 & 3.2 & $1.2 \pm 0.2$ & $0.16_{-0.07}^{+0.06}$ \\
\hline $3 \mathrm{C} 200$ & 0.458 & 83.2 & 44 & 50 & 34 & $26_{-0.4}^{+0.6}$ & $2.6_{-1.0}^{+0.9}$ \\
\hline $3 C 219$ & 0.174 & 33 & 110 & 146 & 3.8 & $5.4_{-0.3}^{+0.4^{+}}$ & $5.2_{-0.5}^{+0.6}$ \\
\hline $3 \mathrm{C} 228$ & 0.552 & 234 & 63 & 99 & 35 & $13 \pm 2$ & $3.2_{-0.9}^{+0.7}$ \\
\hline $3 \mathrm{C} 236$ & 0.0989 & 6.6 & 433 & 662 & 0.1 & - & $0.69 \pm 0.1$ \\
\hline $3 \mathrm{C} 244.1$ & 0.43 & 126 & 44 & 97 & 48.9 & $19_{-2}^{+3}$ & $4.5 \pm 1$ \\
\hline 3C274.1 & 0.422 & 105 & 197 & 275 & 2.5 & $0.9 \pm 0.1$ & $3.7 \pm 0.3$ \\
\hline $3 \mathrm{C} 285$ & 0.0794 & 3.2 & 69 & 66 & 3.6 & $2.2_{-0.2}^{+0.1}$ & $0.56 \pm 0.1$ \\
\hline $3 \mathrm{C} 295$ & 0.462 & 480 & 7.5 & 10.9 & 1890 & - & $183 \pm 4$ \\
\hline $3 \mathrm{C} 326$ & 0.0895 & 7.4 & 324 & 553 & 0.22 & $0.92_{-0.31}^{+0.45}$ & $4.7_{-4.6}^{+4.0}$ \\
\hline $3 \mathrm{C} 330$ & 0.549 & 269 & 48 & 125 & 52.5 & $10.1_{-0.8}^{+0.9}$ & $4.6_{-0.9}^{+0.7}$ \\
\hline 3C 388 & 0.091 & 9.0 & 20.4 & 22.5 & 67.3 & - & $14 \pm 1$ \\
\hline $3 \mathrm{C} 427.1$ & 0.572 & 339 & 44 & 61 & 71 & $78 \pm 4$ & $26 \pm 3$ \\
\hline $3 \mathrm{C} 452$ & 0.0811 & 16.4 & 95 & 106 & 2.4 & $2.8 \pm 0.2$ & $0.79_{-0.07}^{+0.05}$ \\
\hline $3 \mathrm{C} 457$ & 0.428 & 100 & 232 & 367 & 1.5 & $1.8 \pm 0.2$ & $6.1 \pm 1.1$ \\
\hline $6 \mathrm{C} 1132+3439$ & 0.512 & 21.4 & 90 & 176 & 5.7 & $6.9 \pm 0.6$ & $7 \pm 1$ \\
\hline $6 \mathrm{C} 1200+3416$ & 0.53 & 14.8 & 58 & 111 & 8.2 & $17_{-2}^{+1}$ & $8.3 \pm 0.9$ \\
\hline $6 \mathrm{C} 0857+3945$ & 0.528 & 21.9 & 157 & 387 & 2.2 & $1.3_{-0.4}^{+0.8}$ & $2.9_{-2.1}^{+1.6}$ \\
\hline PKS0038+09 & 0.188 & 28 & 57 & 57.4 & 10.8 & $14_{-3}^{+4}$ & $2.7_{-0.8}^{70.6}$ \\
\hline PKS0043-42 & 0.116 & 17.1 & 31 & 96 & 18.4 & $3.7 \pm 0.3$ & $1.3_{-0.4}^{+0.3}$ \\
\hline PKS0213-13 & 0.147 & 17 & 58 & 58.1 & 8.6 & $2.1_{-0.6}^{+0.7}$ & $0.21_{-0.1}^{+0.08}$ \\
\hline PKS0349-27 & 0.0657 & 3.6 & 79 & 160 & 1.6 & $0.63_{-0.1}^{+0.0}$ & $0.3 \pm 0.2$ \\
\hline PKS0945+07 & 0.0863 & 8.2 & 64 & 108 & 2.9 & $2.8_{-0.3}^{+0.4}$ & $1.8_{-0.3}^{+0.2}$ \\
\hline PKS1559+02 & 0.1041 & 13.3 & 84 & 107 & 3.5 & $0.8 \pm 0.1$ & $0.8 \pm 0.2$ \\
\hline PKS2221-02 & 0.0559 & 3.0 & 98 & 163 & 1.3 & $0.92_{-0.08}^{+0.10}$ & $1.4_{-0.2}^{+0.1}$ \\
\hline PKS2356-61 & 0.0963 & 18.7 & 78 & 147 & 4.3 & $2.3 \pm 0.2$ & $1.4_{-0.4}^{+0.3}$ \\
\hline
\end{tabular}

